\section{Inspection Time}

Ronald Cohen

Center for Cognitive Aging and Memory, University of Florida, Gainsville, FL, USA

\section{Definition}

Inspection time refers to the time required during visual processing to make accurate decisions about target stimuli that are presented.

\section{Current Knowledge}

Inspection time is considered to be strongly linked to processing speed. Studies of intellectual functioning have also shown a relationship between inspection time on visual processing paradigms and overall intellectual functioning. In general terms, the term inspection time can be used to describe the duration of visual processing conducted by a particular person on any task requiring a perceptual decision. When subjects are given as much time as needed to inspect a stimulus, they will vary in this duration, though in an uncontrolled experimental context it is difficult to know with certainty whether this reflects the processing speed limitations of the individual or other factors, such as their response bias. To control for this issue, cognitive and visual science researchers use the term more narrowly to refer to the minimal duration of stimulus exposure of visual stimuli for which a high level of accuracy (often $>90 \%$ ) is possible. This duration varies across individuals and the required perceptual tasks.

One of the most common methods for studying inspection time is through the use of visual masking during perceptual judgment. For example, a test may consist of presenting two visual patterns with the task requirement to determine if they are the same or different. The stimuli are presented for varying durations followed by a visual mask to interfere with subsequent shortterm storage. In such a paradigm, inspection time is minimum exposure time that will consistently achieve the accuracy criteria. While a valuable way of assessing information processing speed capacity, this method tends not to be a standard part of clinical neuropsychological assessment at this point in time.

\section{Cross-References}

- Information Processing Speed

- Signal Detection

- Temporal Inhibition 